\title{
AGING AND STEERING CONTROL UNDER REDUCED VISIBILITY CONDITIONS
}

\author{
Bobby Nguyen $^{1}$, Yan Zhuo ${ }^{2}$, \& Rui $\mathrm{Ni}^{1}$ \\ ${ }^{1}$ Wichita State University, Wichita, Kansas, USA \\ ${ }^{2}$ Institute of Biophysics, Chinese Academy of Sciences, Beijing, China \\ Email: rui.ni@wichita.edu
}

\begin{abstract}
Summary: The current study investigated age-related differences in a steering control task under low visibility conditions. Younger and older drivers were presented with displays simulating forward vehicle motion through a 3D scene of random dots on a ground plane. The lateral position of the vehicle was perturbed by a simulated side wind gust according to a sum of sinusoidal functions. The drivers' task was to steer the vehicle to maintain a straight path. The visibility of the driving scene was reduced by reducing the quantity and the quality of the optical flow field. We found that performance decreased when visibility was reduced for both older and younger drivers, with better performance for younger drivers as compared with older drivers. An age-related interaction was also found with deteriorated optical flow information. These results suggest that under reduced visibility conditions, older drivers may have increased accident risk due to decreased ability to successfully steer the vehicle.
\end{abstract}

\section{INTRODUCTION}

Driving at night or in fog requires drivers to process visual information under reduced visibility conditions. Previous research has found that the presence of fog may lead to higher injury and death rates in accidents (Al-Ghamdi, 2007). Driving simulation studies have found evidence of decreased driving performance under simulated fog conditions. Previous studies have shown that fog decreased the judged car following distance for younger drivers (Kang et al., 2008). More importantly, reduced driving performance in fog was more pronounced for older drivers in the car following task (Ni, et al., 2010) and detecting collision events on linear trajectories (Bian et al., 2009).

An important perceptual task during driving is to use visual information to steer the vehicle. Failure to accurately detect changes in the path of motion and make corrections to the vehicle's path of motion could have serious consequences for driver safety. One source of information that has been extensively studied for the perception and control of steering is optical flow---the perspective transformation of the optic array (Gibson, 1966; 1979). Research on optical flow has demonstrated the usefulness of this information for the perception of heading (Warren, Morris, \& Kalish, 1988), the perception of self-motion (Andersen \& Braunstein, 1985), and the perception of egospeed (Larish \& Flach, 1990).

It is well documented in the literature that contrast sensitivity is reduced with increased age (Owsley et al., 1983). Overall contrast of the driving scene is greatly reduced when driving at night or in fog. Previous research has demonstrated age-related decrements in the perception of motion and in the use of optical flow information for the perception of 2D shape (Andersen \& Andersen, 2008) and 3D shape (Andersen \& Atchley, 1995). These results suggest that older 
drivers are likely to have more difficulties in steering control under low contrast conditions than younger drivers because of the reduced visibility of the optical flow field. In the current study, we examined age-related differences in steering control when the optical flow information deteriorates under low visibility conditions.

The simulated deterioration of optical flow information can be either caused by nighttime driving or driving in fog. The reduced visibility can affect the optical flow information in two ways. First, the quantity of optical flow, number of visible dots, is reduced. Second, the quality of optical flow, the contrast of visible dots, is also reduced. Two hypotheses concerning age related differences were examined in the study. Previous research has demonstrated age-related decrements in the perception of motion and in the use of optical flow information for the perception of 2D and 3D shape. We propose that older drivers, as compared to younger drivers, will show a decreased steering performance with reduced quantity of optical flow. We will refer to this hypothesis as the quantity hypothesis. To test this hypothesis, we manipulated the number of dots in the scene (resulting in a decreased quantity of optical flow). If the quantity hypothesis is correct, then age-related differences in steering performance should be more pronounced with smaller as compared to greater amount of dots.

The second hypothesis concerns the contrast of dots in the scene. Previous research has shown age-related decrements in contrast sensitivity. We believe that older drivers may have difficulty processing optical flow with reduced contrast. As a result, older drivers, as compared to younger drivers, may have reduced accuracy in steering control when the optical flow contrast is reduced. We will refer to this hypothesis as the quality hypothesis. To examine this hypothesis we presented optical flow in different contrasts. If older drivers have greater difficulty in using low contrast optical flow information then we predict that older drivers, as compared to younger drivers, will have more steering error when the dots array is presented in low contrast.

\section{EXPERIMENT}

\section{Methods}

Drivers. The drivers were 22 younger (mean age $=20.9$ ) and 17 older (mean age $=78.1$ ) individuals. All drivers were screened for basic cognitive and perceptual ability. All drivers had a minimum of 2 years of driving experience, had normal or corrected to normal vision, and were naïve to the purpose of the experiment. Driver's visual attention was measured using the Useful Field of View (UFOV) task, which comprises a processing speed, divided attention, and selective attention score.

Apparatus. The displays were presented on a 46-inch flat screen LCD monitor with a pixel resolution of 1920 by 1080 at a refresh rate of $60 \mathrm{~Hz}$, controlled by a Windows XP Professional Operating System. The visual angle was approximately $25^{\circ}$ by $15^{\circ}$. Drivers viewed the displays binocularly at a distance of approximately $215 \mathrm{~cm}$ from the screen. A Logitech Driving Force GT steering wheel system was used for steering control. Spring effect, damper effect, and centering spring strength were at 50\%. Angular displacement of the steering wheel was linearly related to the horizontal displacement in the simulation. Drivers were seated in a fixed-base driver's seat with adjustable steering wheel positioning with an eye height of approximately $115 \mathrm{~cm}$. Drivers 
viewed the displays in a darkened room and were given at least 5 minutes for dark-adaptation prior to the experiment.

Stimuli. The displays simulated driving through a 3D array of dots located on a ground plane. The simulated speed was $72 \mathrm{~km} / \mathrm{h}$ (45 M/h). The dimensions of the space were $400 \mathrm{~m}$ (width) by $150 \mathrm{~m}$ (depth), with the simulated eye position of the driver at $1.6 \mathrm{~m}$ above the ground. The horizontal position of the dots pattern was perturbed according to a sum of 3 prime sine-wave functions. Amplitudes and frequencies were selected to provide equal energy in the Fourier domain. The three frequencies used were $0.083,0.161$, and $0.216 \mathrm{~Hz}$. The amplitudes for these frequencies were 17.51, 6.74, and $9.02 \mathrm{~m}$, respectively. The first two phases of the sine-wave function were randomized across trials. The last phase was determined such that the output of the sum of the three sine-wave functions was zero at the beginning of each trial. The scene consisted either of 25 or 125 randomly positioned dots to form the ground plane. The dots were randomly positioned at the beginning of each trial, and then the viewpoint moved forward. Dots that moved out of the viewport would be randomly repositioned at the far end of the ground plane.

The background had an average luminance of $16 \mathrm{~cd} / \mathrm{m}^{2}$. Luminance of the dots was 21.65, 24.00, and $26.67 \mathrm{~cd} / \mathrm{m}^{2}$, which generated a Michelson contrast of $0.15,0.20$, and 0.25 , for the low, medium, and high contrast condition, respectively. All drivers reported during debriefing that they were able to see the dots in all conditions.

Design. The independent variables for the steering control task were dot number in the optic flow field (25 or 125), contrast level (low, medium, and high), and age group (younger and older). Age group was treated as a between-subjects variable. All other variables were treated as withinsubject variables. Two repetitions for each of the six conditions generated 12 trials for each driver. The order of the trials for each driver was randomized individually.

Procedure. Drivers first completed the visual acuity and contrast sensitivity measurements, and then proceeded to complete the UFOV tasks. After completing the visual tasks, drivers were moved to the simulator to complete the steering control task. The driver's seat and steering wheel position were adjusted to suit their comfort.

Drivers were told that the display was comparable to driving down a straight roadway with lateral wind gusts perturbing their position on the roadway. In addition, they were told that their task was to correct their position on the roadway such that they maintained driving along a straight path. Each trial began with a 10-second straight path period, in which input from steering wheel and perturbation were disabled. This was done to set up the perception of straight path for the drivers. At the end of the 10 seconds, a beep sounded to notify the drivers that they need to steer the wheel to null out the perturbation. At the end of each trial, a blank screen indicated that a break can be taken. When the driver was ready for the next trial, they were instructed to press the shift lever to advance. Drivers were given at least two practice trials in which display was in maximum contrast (black background and white dots) and amplitude of the sine-wave functions were decreased by $50 \%$. Once the drivers understood the task they were given the experimental trials. 


\section{RESULTS}

Steering performance was assessed by calculating RMS (root means square) steering error in relation to perturbation forces for each driver on each trial in each condition. A higher RMS score represents a greater deviation from the straight driving path indicating poorer driving performance. It was observed in some trials, due to the accumulation of displacement over a boundless driving space, drivers could not return to the original straight path after a certain amount of time. Therefore, an algorithm was developed to distinguish periods of a trial in which a participant became "lost”. For each trial, an overall RMS score was computed for all data points. Then, for each individual data point, a RMS score was computed for a 31-frame period (15 preceding, current data point, and 15 proceeding). The data point was deleted if the current RMS score was greater than the overall RMS score (about 37\% data was dropped for both younger and older drivers). A final RMS score was computed for the remaining data points. Scores were averaged across repetitions, resulting in six scores (one for each condition) for each participant.
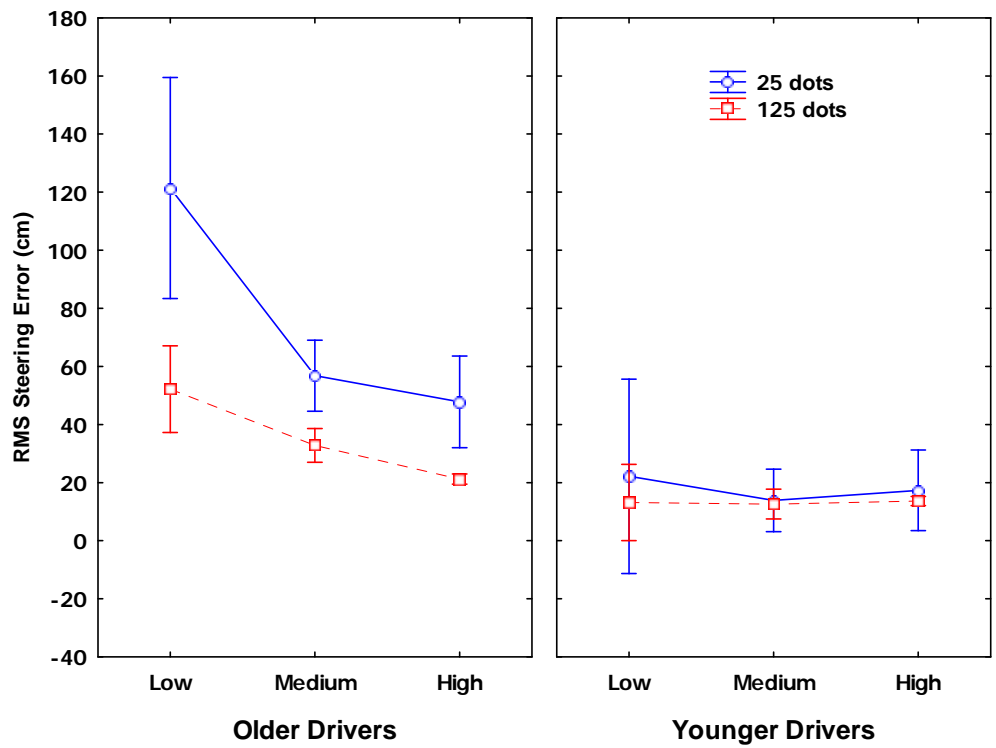

Figure 1. The combined results of steering error as a function of dots contrast for older and younger drivers

Table 1. Mean (SD) RMS scores for steering control task

\begin{tabular}{ccccccc}
\hline & \multicolumn{3}{c}{ Low dot density (25) } & \multicolumn{3}{c}{ High dot density (125) } \\
\cline { 2 - 7 } & Low & Medium & High & Low & Medium & High \\
& Contrast & Contrast & Contrast & Contrast & Contrast & Contrast \\
\hline Younger & $22.17(12.14)$ & $13.91(2.73)$ & $17.36(7.06)$ & $13.19(1.87)$ & $12.62(1.55)$ & $13.69(3.11)$ \\
Older & $121.42(116.91)$ & $56.84(37.70)$ & $47.83(48.12)$ & $52.18(46.14)$ & $32.83(17.91)$ & $21.21(4.15)$ \\
\hline
\end{tabular}

Table 1 shows the mean performance scores by condition and age group. A three-way mixed design analysis of variance (ANOVA) was conducted to evaluate the effect of dot density, contrast level, and age on driving performance. Younger drivers had significantly less steering error than older drivers $(F(1,37)=29.81, p<.01)$. Both older and younger drivers had greater steering error with a reduced number of dots $(F(1,37)=21.78, p<.01)$ and reduced contrast of 
the dots $(F(2,36)=10.0, p<.01)$ in the flow field. The density by age interaction effect was significant $(F(1,37)=13.64, p<.01)$, and the contrast by age interaction effect was significant $(F(2,36)=10.77, p<.01)$. The density by contrast interaction effect was significant, $(F(2,74)=$ $3.95, p<.05)$. The three-way interaction among density, contrast, and age was not significant $(F(2,36)=1.27, p=.30)$. These results, as illustrated in Figure 1, showed that older drivers had consistent great steering error as compared to younger drivers especially when optical flow information decreased in either quantity or quality.

Table 2. Correlations between UFOV scores and driving conditions

\begin{tabular}{ccccccc}
\hline & \multicolumn{3}{c}{ Low dot density (25) } & \multicolumn{3}{c}{ High dot density (125) } \\
\cline { 2 - 7 } & $\begin{array}{c}\text { Low } \\
\text { Contrast }\end{array}$ & $\begin{array}{c}\text { Medium } \\
\text { Contrast }\end{array}$ & $\begin{array}{c}\text { High } \\
\text { Contrast }\end{array}$ & $\begin{array}{c}\text { Low } \\
\text { Contrast }\end{array}$ & $\begin{array}{c}\text { Medium } \\
\text { Contrast }\end{array}$ & $\begin{array}{c}\text { High } \\
\text { Contrast }\end{array}$ \\
\hline Processing speed & $.32^{*}$ & 0.0 & .13 & 0.0 & .06 & .18 \\
Divided attention & $.52^{* *}$ & $.50^{* *}$ & $.36^{*}$ & $.48^{* *}$ & $.32^{*}$ & $.54^{* *}$ \\
Selective attention & $.55^{* *}$ & $.55^{* *}$ & .30 & $.43^{* *}$ & $.48^{* *}$ & $.71^{* *}$ \\
\hline
\end{tabular}

* Correlation is significant at the 0.05 level (2-tailed)

** Correlation is significant at the 0.01 level (2-tailed)

Table 3. Correlations between contrast sensitivity and driving conditions

\begin{tabular}{ccccccc}
\hline & \multicolumn{3}{c}{ Low dot density (25) } & \multicolumn{3}{c}{ High dot density (125) } \\
\cline { 2 - 7 } & $\begin{array}{c}\text { Low } \\
\text { Contrast }\end{array}$ & $\begin{array}{c}\text { Medium } \\
\text { Contrast }\end{array}$ & $\begin{array}{c}\text { High } \\
\text { Contrast }\end{array}$ & $\begin{array}{c}\text { Low } \\
\text { Contrast }\end{array}$ & $\begin{array}{c}\text { Medium } \\
\text { Contrast }\end{array}$ & $\begin{array}{c}\text { High } \\
\text { Contrast }\end{array}$ \\
\hline Binocularly & -.14 & $-.39^{*}$ & -.13 & $-.60^{* *}$ & $-.33^{*}$ & $-.37^{*}$ \\
\hline
\end{tabular}

* Correlation is significant at the 0.05 level (2-tailed)

** Correlation is significant at the 0.01 level (2-tailed)

Table 4. Correlations between visual acuity and driving conditions

\begin{tabular}{|c|c|c|c|c|c|c|}
\hline & \multicolumn{3}{|c|}{ Low dot density (25) } & \multicolumn{3}{|c|}{ High dot density (125) } \\
\hline & $\begin{array}{c}\text { Low } \\
\text { Contrast } \\
\end{array}$ & $\begin{array}{l}\text { Medium } \\
\text { Contrast } \\
\end{array}$ & $\begin{array}{c}\text { High } \\
\text { Contrast }\end{array}$ & $\begin{array}{c}\text { Low } \\
\text { Contrast } \\
\end{array}$ & $\begin{array}{l}\text { Medium } \\
\text { Contrast } \\
\end{array}$ & $\begin{array}{c}\text { High } \\
\text { Contrast }\end{array}$ \\
\hline Binocularly & $-.38 *$ & $-.43 * *$ & -.24 & $-.47 * *$ & $-.43 * *$ & $-.55 * *$ \\
\hline
\end{tabular}

* Correlation is significant at the 0.05 level (2-tailed)

** Correlation is significant at the 0.01 level (2-tailed)

Correlation coefficients were computed between the visual assessment scores and six steering control task conditions. The Holm's sequential Bonferroni approach was used to control for Type I error across the correlations. The results of the correlational analyses between the driving conditions and UFOV scores, contrast sensitivity, and visual acuity are presented in Tables 2, 3, and 4, respectively. Further analysis showed correlations among the processing speed and divided attention were moderately correlated, $\mathrm{r}(39)=.39, p<.05$. Divided attention and selective attention were strongly correlated, $\mathrm{r}(39)=.76, p<.01$.

\section{DISCUSSION}

In this study, we examined age-related differences in the use of optical flow information when performing a steering control task under reduced visibility conditions. We predicted that when visibility was reduced older drivers would show greater decrements because of decreased 
contrast sensitivity and decrements in processing optical flow. The results provide evidence supporting both the quantity and quality hypothesis. Specifically, when deteriorated optical flow was presented, either with reduced number of dots or with reduced contrast, older drivers showed greater decrements in steering performance as compared with younger drivers. Considered together, these results suggest that under low visibility conditions older drivers may be subject to an increased accident risk due to a decreased ability to process optical flow information for steering control. The quantity of optical flow information plays a more important role, as compared to the quality of optical flow, in determining steering control performance for older drivers. This result suggests that a potential intervention for age-related increased accident risks at night can be implemented by placing more reflectors on the road, which generates more optical flow information for steering control.

Past research (Ball, et al., 1993; Owsley, et al., 1991) has suggested different assessments that are better predictors of driving performance and crash risk than visual acuity. The correlational analysis results in the current study support these findings. Specifically, the highest loading factors for contrast sensitivity and UFOV tasks were -.73 and -.71, respectively, as compared to .64 for visual acuity.

The current study adds to the growing body of literature showing that the decreased ability in processing perceptual information accompanying aging may lead to increased risk for older drivers (e.g., Ni et al. 2007), especially under low visibility conditions which result from driving at night or under inclement weather conditions (e.g., in fog). An important issue for future research is whether this decrement can be minimized or even reversed through training. Andersen et al. (2010) found that training with subthreshold stimuli improved older observers when performing a texture discrimination task. Richards et. al. (2006) found that older observers could improve their performance in divided attention through repeated training with the UFOV task, and that this improvement was retained for up to 3 months. Similarly, improvements in contrast sensitivity through training were found in a most recent study (Phan and Ni, 2011). An interesting topic for future research would be to investigate whether older drivers can improve steering control performance through the use of training techniques.

\section{ACKNOWLEDGMENT}

This research was supported by WSU Gridley \& Hoover grant and NNSF of China grant 90820307.

\section{REFERENCES}

Al-Ghamdi, A. S. (2007). Experimental evaluation of fog warning system. Accident Analysis and Prevention, 39, 1065-1072.

Andersen, G. J., \& Atchley, P. A. (1995). Age related differences in the detection of 3D surfaces from optic flow, Psychology and Aging, 10, 650-658.

Andersen, G. J., \& Braunstein, M. L. (1985). Induced self-motion in central vision. Journal of Experimental Psychology: Human Perception \& Performance, 11, 122-132.

Andersen, G. J., and Ni, R. (2008). Aging and visual processing: declines in spatial not temporal integration, Vision Research, 48, 109-118. 
Andersen, G. J., Ni, R., Bower, J.D., Watanabe, T. (2010). Perceptual learning, aging, and improved visual performance in early stages of visual processing, Journal of Vision, 10(13):4, $1-13$.

Ball, K., Owsley, C., Sloane, M.E., Roenker, D.L., \& Bruni, J.R. (1993). Visual attention problems as a predictor of vehicle crashes among older drivers. Investigative Ophthalmology \& Visual Science, 34(11), 3110-3123.

Bian, Z., Ni, R., Guindon, A., \& Andersen, G. J. (2007). Aging and the detection of collision events in fog. Proceedings of the Fifth International Driving Symposium on Human Factors in Driver Assessment, Training and Vehicle Design, 69-75.

Gibson, J. J. (1966). The senses considered as perceptual systems. Boston: Houghton Mifflin.

Gibson, J. J. (1979). The ecological approach to visual perception Boston: Houghton Mifflin.

Kang, J. J., Ni , R., \& Andersen, G. J. (2008). The effects of reduced visibility from fog on car following performance. Transportation Research Record: Journal of the Transportation Research Board, 2069, 9-15.

Larish, J. F., \& Flach, J. M. (1990). Sources of optical information useful for perception of speed of rectilinear self-motion. Journal of Experimental Psychology: Human Perception \& Performance, 16, 295-302.

Owsley, C., Ball, K., Sloane, M., Roenker, D.L., \& Bruni, J.R. (1991). Visual perceptual/cognitive correlates of vehicle accidents in older drivers. Psychology and Aging, 6, 403-415.

Owsley, C., Sekuler, R., \& Siemsen, D. (1983). Contrast sensitivity throughout adulthood. Vision Research, 23, 689-699.

Phan, M., \& Ni, R., (2011). Training older adults' to improve their contrast sensitivity: A possible or impossible task. Submitted.

Ni, R., Kang, J., \& Andersen, G. J. (2007). Age-related driving performance: effect of fog under dual-task conditions. Proceedings of the Fourth International Driving Symposium on Human Factors in Driver Assessment, Training and Vehicle Design, 365-370.

Ni, R., Kang, J., Andersen, G. J. (2010). Age-related declines in car following performance under simulated fog conditions, Accident Analysis \& Prevention, 42(3), 818-826.

Richards, E., Bennett, P. J., Sekuler, A. B. (2006) Age related differences in learning with the useful field of view. Vision Research. 46, 4217-4231.

Warren, W. H., Morris, M. W., \& Kalish, M. (1988). Perception of translational heading from optical flow. Journal of Experimental Psychology: Human Perception and Performance, 14, 646-660. 